\title{
Assessment of COVID- 9 Vaccine Acceptance among Health Care Workers and General Population - A Cross-Sectional Survey
}

\author{
Abinaya E, Maignana Kumar R, Ruckmani A, Arunkumar R \\ Department of Pharmacology, Chettinad Hospital \& Research Institute, Chettinad Academy of Research and Education (CARE), \\ Kelambakkam, Chennai, Tamil Nadu, India.
}

DOI: https://doi.org/10.24321/0019.5138.202130

\section{I $\quad \mathbf{N} \quad \mathbf{F} \quad \mathbf{O}$}

\section{Corresponding Author:}

Arunkumar R, Department of Pharmacology, Chettinad Hospital \& Research Institute, Chettinad Academy of Research and Education (CARE), Kelambakkam, Chennai, Tamil Nadu, India.

E-mail Id:

drarunvp@gmail.com

Orcid Id:

https://orcid.org/0000-0002-7657-7530

How to cite this article:

Abinaya E, Maignana Kumar R, Ruckmani A, Arunkumar R. Assessment of COVID-19 Vaccine Acceptance among Health Care Workers and General Population - A Cross-Sectional Survey. J Commun Dis. 2021; 53(2): 82-88.

Date of Submission: 2021-06-11

Date of Acceptance: 2021-06-14

\section{$\begin{array}{llllllll}\mathbf{A} & \mathbf{B} & \mathbf{S} & \mathbf{T} & \mathbf{R} & \mathbf{A} & \mathbf{C} & \mathbf{T}\end{array}$}

Introduction: This cross-sectional survey was conducted to estimate the proportion of participants among health care workers and general population who are willing to get vaccinated against COVID-19 and those who are not, and to identify the reasons for vaccine hesitancy among the participants.

Methods: Health care workers of a tertiary care hospital and the general population residing in the nearby areas of the tertiary care hospital were predominantly included in the study. A specific questionnaire was designed, validated, and administered to the participants after obtaining consent.

Result: The responses were collected using Google Form and compiled in an excel sheet for analysis. The difference in opinion with regard to acceptance of the COVID-19 vaccine was statistically analysed using Chisquare test. The vaccine acceptance rate was high among the general population $(70.2 \%)$ as compared to health care workers $(56.7 \%)(p=$ 0.0006 , highly significant). The remaining proportions of participants (43.3\% of health care workers and $29.8 \%$ of general population) were hesitant to receive the COVID-19 vaccine.

Conclusion: The reasons for vaccine hesitancy were fear of harmful effects, doubtful efficacy, lack of transparency in clinical trials, and fast track vaccine development.

Keywords: COVID-19 vaccine, Acceptance, Hesitancy, Health Care Workers, General Population

\section{Introduction}

The outbreak of COVID-19, caused by severe acute respiratory syndrome coronavirus 2 (SARS-CoV-2), started in Wuhan, China in December 2019. Subsequently, WHO announced it as a global pandemic on March 11, 2020. Till now, COVID-19 has infected over 113,315,218 people and caused mortality in 2,517,964 people across the world (WHO). ${ }^{1}$ There is no effective treatment for COVID-19 till date and it is difficult to prevent the spread, despite adopting quarantine and lockdowns, social distancing and personal hygiene measures like wearing face masks, using hand sanitisers, etc. There is an intense effort worldwide to 
develop a safe and effective vaccine for COVID-19. Currently, more than 180 vaccine candidates are under various stages of development for SARS CoV-2. ${ }^{2}$ Among the vaccines in development, US-FDA has given emergency use authorisation for two vaccines - Pfizer-BioNTech COVID-19³ on December 11 and Moderna COVID-194 vaccine on December 18, 2020. In India, Covishield developed by Serum Institute of India, and Covaxin developed by Bharat Biotech were approved for restricted emergency use on January 3, 2021. Though the governments are intensively working on bringing out the vaccines, there is some reluctance among the public and health care professionals in getting vaccinated against COVID-19. A few surveys reported that the general population and even some doctors are hesitant to get vaccinated since they are not fully aware of the potential benefits and risks of the vaccines. A survey conducted among the US general population showed that $57.6 \%$ of participants wanted to get vaccinated, $31.6 \%$ were not sure, and $10.8 \%$ did not intend to be vaccinated. ${ }^{5}$ In a vaccine hesitancy survey conducted by the Oxford University in the UK among the adult population, it was found that $72 \%$ were willing to be vaccinated, $16 \%$ were very unsure about receiving a COVID-19 vaccine, and another $12 \%$ were likely to delay or avoid getting the vaccine. ${ }^{6}$ In an online study conducted by the Coordinator of the Chennai Declaration and Infectious Diseases Consultant at Apollo Hospital, Chennai, among 1424 health professionals, $45 \%$ were willing to take the COVID-19 vaccine as soon as it became available. 55\% wanted to either defer the vaccination or had not decided. About one-tenth of the participants responded that they would never take the vaccine. ${ }^{7}$ Hence, we planned this survey to find out the vaccine hesitancy and the factors associated with it among doctors, other health care workers, and the general population in this present scenario where we have options to receive COVID-19 vaccines, either Covishield or Covaxin. The objective of the study was to estimate the proportion of participants among the health care workers and general population who were willing to get vaccinated against COVID-19, compare the proportion among the various study groups and strata, and identify the reasons for vaccine hesitancy among the participants in various study groups and strata.

\section{Materials and Methods}

The survey was initiated after obtaining approval from the Institutional Human Ethics Committee. It was a crosssectional survey wherein the participants were requested to respond to a set of questions designed to capture the relevant data on vaccine hesitancy. Doctors, nurses, pharmacists, technicians, and other paramedical staff in Chettinad Hospital and Research Institute and other nearby hospitals, and general population - age more than 18 years, and residing in the nearby areas of Chettinad Hospital and Research Institute were the participants included in this study.
The plan was to enrol a total of 500 participants for this survey with 250 participants among healthcare workers and 250 among the general population. The statistical logic was that 188 participants were required per group for the assumed proportion of 55\% vaccine hesitancy (based on the Chennai study ${ }^{7}$ ), and precision of $7.5 \%$ (two-tailed) at $95 \%$ significance. This number was increased to 250 per group to augment the validity of the outcome. However, efforts were made to enrol as many participants as possible over and above 250 per group. The questionnaire was prepared as a Google Form and was validated in a pilot study by administering it to 10 volunteers who would not participate in the actual survey. After validation, the questionnaire was circulated through e-mail and other online telecommunication systems to the potential participants. Informed consent was obtained from the participants before they started answering the questions. The questionnaire was designed in such a way that if the participants consented to participate in the survey by selecting 'yes' for the question "Are you willing to participate in this online survey on the COVID-19 vaccine acceptance among health care workers and general population?", further questions would appear for them. If they answered 'no', the participants would not get further questions. The initial part of the questionnaire was framed to record the demographic details (age, gender, profession) of the participants. The nature of the profession was assessed with a 'closed' question having two options,' health care professionals' and 'general population'. If the participant was a health care professional, he/ she had to answer a semi-open question to select the category such as doctor, nurse, pharmacist, technician, and others. Further details such as the speciality in which the health care professional was working and whether he/ she was involved in treating COVID-19 patients/ working in COVID-19 wards were collected. Except for the above-mentioned details for health care workers, the other questions were common for both health care workers and general population. In order to assess the knowledge about the COVID-19 vaccines among the participants, a closed question 'Are you aware of the potential COVID-19 vaccines?' with 'yes' or 'no' options was given. If the participant was aware of the vaccines, then a semi-open question to know about the sources from which they got information about vaccines, followed. The options given for the potential sources of information were television, newspaper, internet and family physician. The next part of the questionnaire had two closed questions to assess the attitude among the participants about COVID-19 vaccines. The first question was 'Do you think vaccination will prevent you from getting COVID-19?' with options given in a 4-point Likert scale - strongly agree, agree, disagree, and strongly disagree. The other question was 'Do you think there is adequate safety information about COVID-19 vaccines available?' with dichotomous options -'yes' and 'no'. The 
subsequent section of the questionnaire had two questions to know about the acceptance of COVID-19 vaccine. The willingness in getting vaccinated against COVID-19 was assessed using a 4-point Likert scale - strongly agree, agree, disagree, and strongly disagree. Among the respondents who accepted to take the vaccine, we assessed how soon after vaccine availability, they were ready to get vaccinated using the following time intervals - a) Immediately after availability, b) within 1 month, c) 1 to 3 months, d) 3 to 6 months and e) not sure. Finally, a semi-open question was given to know if the participants had any concern about the vaccine with regard to its safety, efficacy, cost, and other factors. The vaccine acceptance was calculated by combining the number of participants who 'strongly agree' and 'agree', and vaccine hesitancy by obtained by combining the number of participants who opted for 'strongly disagree', and 'disagree'. The survey tool was approved by the Ethics Committee on 05/01/2021 and the survey was conducted from $06 / 01 / 2021$ to $15 / 01 / 2021$. The responses from the participants were automatically converted into an excel sheet from the submission of Google Forms by the study participants. The data were statistically analysed with all the variables expressed as frequencies and percentages. The difference in responses between the health care professionals and general population was compared using Chi-square test for categorical data and unpaired t-test for quantitative data.

\section{Results}

A total of 680 responses were received. Among them, 65 were not willing to participate in the survey and the remaining participants responded to the questions. Based on the profession, the participants were divided into 2 groups - health care workers and general population. There were 333 healthcare workers and 282 from general population. The mean age ( \pm standard deviation) of the health care workers was $35.5( \pm 11.58$ ) years and general population was 35.6 ( \pm 11.68 ) years. The two groups did not differ in terms of age ( $p=0.15$, unpaired t-test). The number of males and females in the health care workers and the general population groups is shown in Figure 1. There were a greater number of females than males in the health care group and the opposite was true for the general population.

The health care workers who participated in the survey were doctors, nurses, pharmacists, lab technicians, and others like medical representatives, clinical research coordinators, physician assistants, etc. Overall, the doctors constituted $50.1 \%$ of total healthcare workers followed by nurses at $35.1 \%$. It was observed that approximately about $90 \%$ of the participants in both groups were aware of the potential COVID-19 vaccines. 299 healthcare workers (89.78\%) and 260 general population participants (92.19\%) expressed that they were aware of the COVID-19 vaccines. Among the sources from which the participants came to know about the COVID-19 vaccines, internet was the most common source (68.8\%), followed by television (58.1\%), newspaper $(37.7 \%)$, family physician (16.9\%), and other sources $(8.3 \%)$. The other sources mentioned by the participants were research papers, social media, webinars, friends, and peer group discussions at workplace.

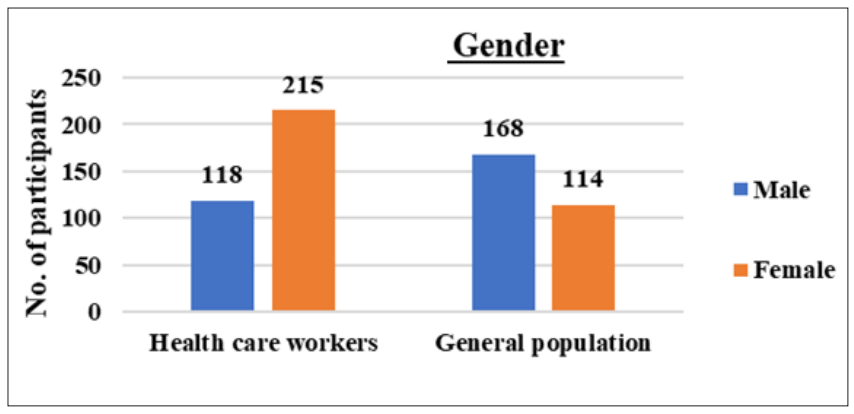

Figure I.Gender-wise Distribution of Study Participants

The responses for the question regarding the opinion of the participants whether the vaccination will prevent them from COVID-19 were collected in 4-point Likert scale as strongly agree, agree, disagree, and strongly disagree. The number of participants in each category is shown in Table 1.

About $75.4 \%$ of health workers and $84.7 \%$ of general population believe (strongly agree + agree) that the vaccination would protect them from COVID-19. There is no significant difference in the proportion of participants in "strongly agree", "agree", and "strongly disagree" categories between healthcare workers and general population. The category 'disagree' had more number of health care workers compared to the general population and the difference was statistically significant too. $60.98 \%$ of the participants (375 out of the total 615 participants) were of the opinion that there was no adequate safety information about the vaccine and the same has been shown in Figure 2, groupwise. The opinion about safety of the vaccine is the same among healthcare workers and general population which did not significantly differ between the groups $(p=0.97$, Chi-square test).

The willingness to receive the vaccine was analysed in three different ways as follows:

- Comparison of vaccine acceptance between health care workers and general population

- Vaccine acceptance among health care workers involved in treating COVID-19 patients and not involved in treating COVID-19 patients

- Vaccine acceptance among participants who had a personal history or family history of COVID-19 infection and those who do not have prior personal/ family history of COVID-19 infection 
Table I.Responses of Study Participants for the question - Do You think Vaccination will prevent You from getting COVID- I9?

\begin{tabular}{|c|c|c|c|c|}
\hline Group & Strongly Agree & Agree & Disagree & Strongly Disagree \\
\hline Health care workers & $39(11.7 \%)$ & $212(63.7 \%)$ & $68(20.4 \%)$ & $14(4.2 \%)$ \\
\hline General population & $48(17.02 \%)$ & $191(67.7 \%)$ & $39(13.8 \%)$ & $4(1.4 \%)$ \\
\hline $\begin{array}{c}\text { Statistics (Chi-square } \\
\text { test) }\end{array}$ & $\begin{array}{c}\mathrm{P}=0.06(\text { not } \\
\text { significant) }\end{array}$ & $\begin{array}{c}\mathrm{P}=0.29(\mathrm{not} \\
\text { significant) }\end{array}$ & $\begin{array}{c}\mathrm{P}=0.03 \\
\text { (significant) }\end{array}$ & $\begin{array}{c}\mathrm{P}=0.07 \\
\text { (not significant) }\end{array}$ \\
\hline Total & $87(14.1 \%)$ & $403(65.6 \%)$ & $107(17.3 \%)$ & $18(2.9 \%)$ \\
\hline
\end{tabular}

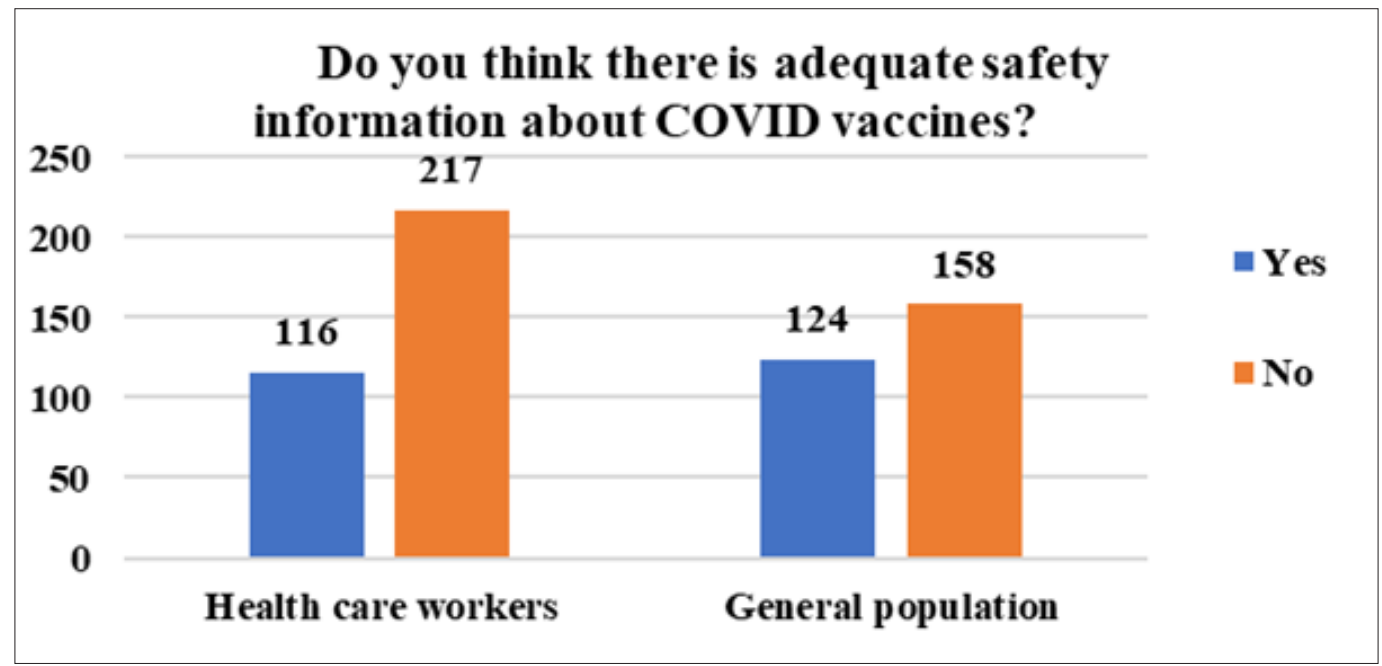

Figure 2.Responses of Study Participants for the question - Do You think there is Adequate Information about COVID- I 9 Vaccines?

These sub-group analyses were carried out so as to understand the factors influencing their decision to receive vaccines. Chi-square test was used to determine the statistical significance among the sub-groups. A significantly higher proportion of general population strongly agreed for vaccination in comparison with health care workers. The proportion of health care workers who disagreed and strongly disagreed for vaccination is significantly high compared to the general population. Yet, $51.1 \%$ of the general population and $45.3 \%$ of the health care workers agreed for vaccination and the difference was not statistically significant. The vaccine acceptance rate among health care workers was $56.7 \%$ and among general population, it was $70.2 \%$ (Chi-square test, $p=0.0006$, highly significant). The relevant details are shown in Table 2. The vaccine hesitancy rate (disagree and strongly disagree) was 43.3\% among the health care workers and $29.8 \%$ among general population. The difference was highly significant (Chi-square test, $\mathrm{p}=0.0005$ ).

When the data among healthcare workers were categorised in terms of those who were involved in COVID-19 care and those who were not, there was no statistically significant difference observed in any of the 4-point Likert scale strongly agree, agree, disagree, and strongly disagree (Table 3).

Table 2.Responses of Study Participants for the question - Will You take the Vaccine as soon as it becomes Available in the Market?

\begin{tabular}{|c|c|c|c|c|}
\hline Group & Strongly Agree & Agree & Disagree & Strongly Disagree \\
\hline Health care workers & $38(11.4 \%)$ & $151(45.3 \%)$ & $100(30.1 \%)$ & $44(13.2 \%)$ \\
\hline General population & $54(19.1 \%)$ & $144(51.1 \%)$ & $62(22 \%)$ & $22(7.8 \%)$ \\
\hline $\begin{array}{c}\text { Statistics } \\
\text { (Chi-square test) }\end{array}$ & $\begin{array}{c}\mathrm{P}=0.007 \\
\text { (significant) }\end{array}$ & $\begin{array}{c}\mathrm{P}=0.15 \\
\text { (not significant) }\end{array}$ & $\mathrm{P}=0.02$ (significant) & $\begin{array}{c}\mathrm{P}=0.04 \\
\text { (significant) }\end{array}$ \\
\hline Total & $92(14.9 \%)$ & $295(47.9 \%)$ & $162(26.3 \%)$ & $66(10.7 \%)$ \\
\hline
\end{tabular}


Table 3.Health Care Workers involved in COVID- I 9 Care and Vaccine Acceptance

\begin{tabular}{|c|c|c|c|c|}
\hline $\begin{array}{c}\text { Health Care Workers (Involved in } \\
\text { Treating CoVID-19 Patients) }\end{array}$ & Strongly Agree & Agree & Disagree & Strongly Disagree \\
\hline Yes (177) & 25 & 77 & 50 & 25 \\
\hline No (156) & 13 & 74 & 50 & 19 \\
\hline $\begin{array}{c}\text { Statistics } \\
\text { (Chi-square test) }\end{array}$ & $\begin{array}{c}\mathrm{P}=0.06 \\
\text { (not significant) }\end{array}$ & $\begin{array}{c}\mathrm{P}=0.54 \\
\text { (not significant) }\end{array}$ & $\begin{array}{c}\mathrm{P}=0.5 \\
\text { (not significant) }\end{array}$ & $\begin{array}{c}\mathrm{P}=0.57 \\
\text { (not significant) }\end{array}$ \\
\hline
\end{tabular}

82 respondents among the health care workers and 56 among the general population had a past history of COVID-19 infection or a positive family history. There was no significant difference in vaccine acceptance among the participants with and without a history of COVID-19 infection in the past, in both, the health worker group ( $p=0.91$, Chi-square test) and general population ( $p=$ 0.22 , Chi-square test). With regard to the question of how soon the participants would want to get vaccinated once the vaccines are made available, there was no significant difference between healthcare workers (Figure 3) and general population (Figure 4).

\section{Concerns about Vaccine}

When the participants who were willing to get vaccinated were asked if they had any concerns about the vaccine, $62.53 \%$ responded that they were not sure whether the vaccine will protect them from COVID-19, followed by concerns about harmful effects (53.85\%), cost (25.26\%), and other reasons (12.67\%). The other reasons quoted were doubtful efficacy, lack of transparency regarding clinical trials, and fast track development of the vaccine (Figure 5).

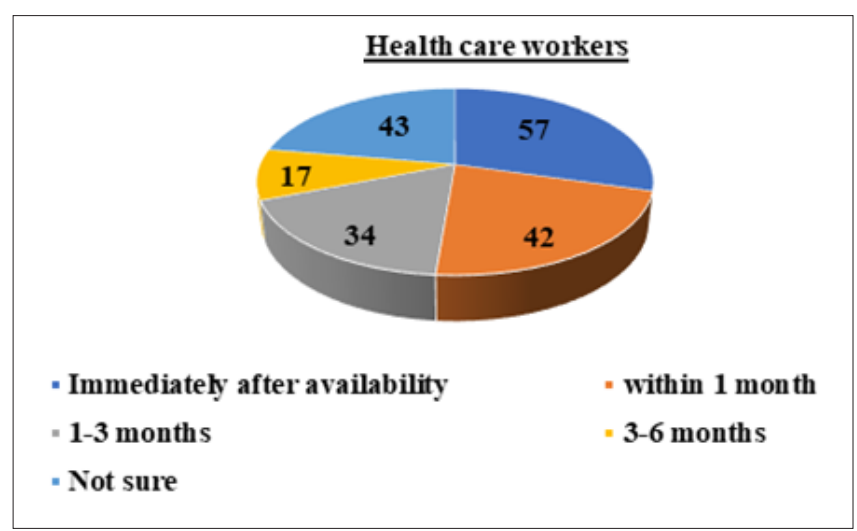

Figure 3.Responses of Health Care Workers for the question - How soon after the Vaccine becomes Available, would You be Ready to get Vaccinated? Among those who did not want to get vaccinated, the primary concern was fear of the harmful effects of the vaccine (67.54\%). $35.53 \%$ of the participants were of the opinion that the vaccine would not protect them from COVID-19 infection, $18.86 \%$ felt that the risk of acquiring COVID-19 infection was low, $8.77 \%$ were concerned about the vaccine cost and the remaining (26.75\%) mentioned other reasons such as the vaccine was approved only for emergency use, limited clinical trials, development of vaccine in a very short period, no transparency about clinical trials, and changing strains of SARS-CoV-2 (Figure 6).

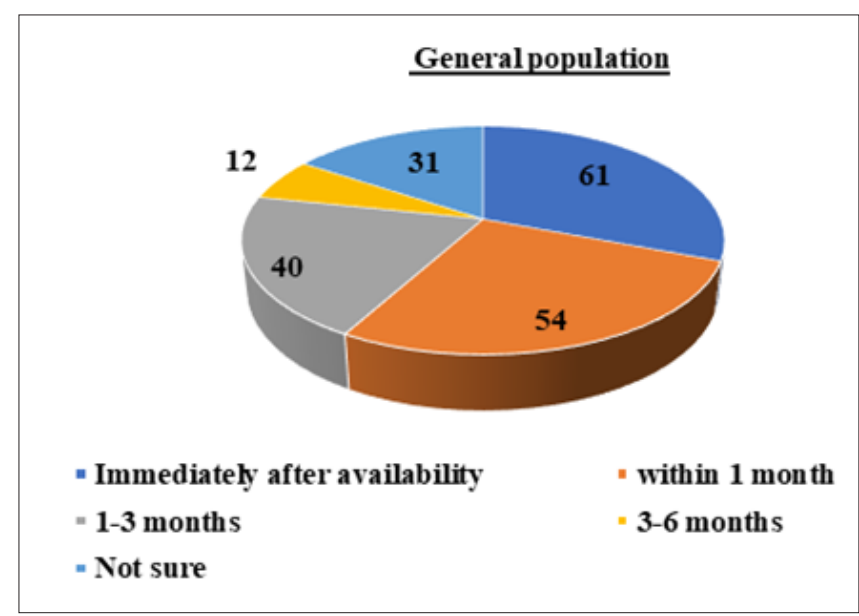

Figure 4.Responses of General Population for the question - How soon after the Vaccine becomes Available, would You be Ready to get Vaccinated?

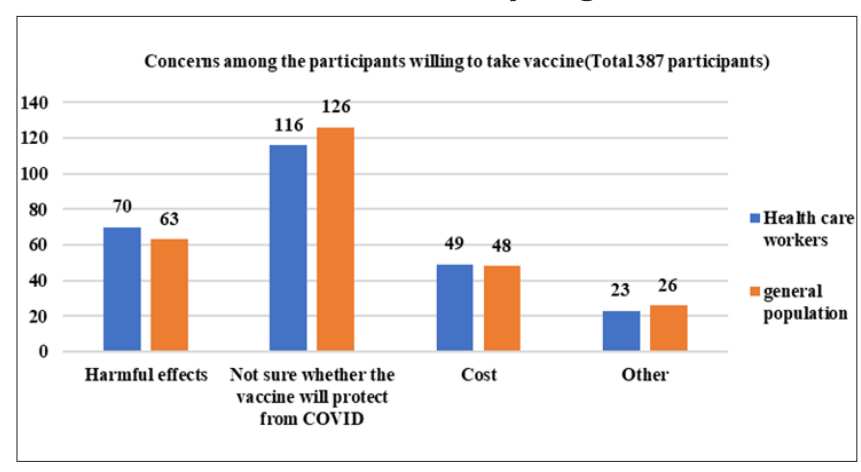

Figure 5.Concerns among the Participants who were willing to take the Vaccine 


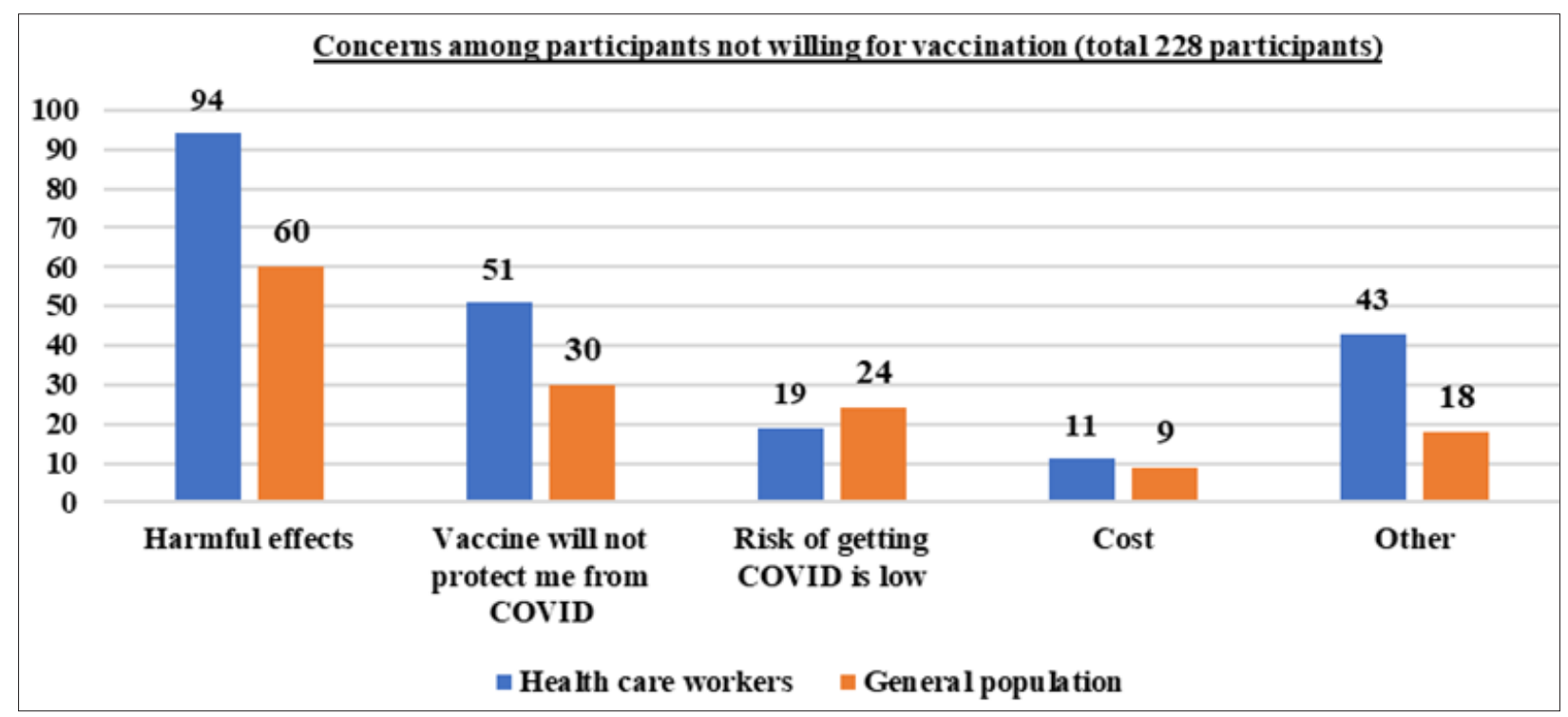

Figure 6.Concerns among the Participants who were not willing to take the Vaccine

\section{Discussion}

This survey was done to assess the COVID-19 vaccine acceptance and hesitancy among the health care workers and the general population. The government of India is intensively working on the immunisation programme against COVID-19 and the roll-out of vaccines has already started for the front-line health workers, geriatric population, and population aged 45 to 59 years with co-morbidities in the country.

It was observed that more than $90 \%$ of the respondents were aware of the COVID-19 vaccines through internet, television, newspaper, etc. About $75.4 \%$ of the health care workers and $84.7 \%$ of the general population believe that the vaccination will protect them from COVID-19 infection. Regarding the safety of the vaccine, $65.2 \%$ of the health care workers and $56.02 \%$ of general population feel that there was no adequate safety information about the vaccine. This could be due to the inadequate information available in the literature and other public domains that strongly support the vaccine's safety. Further, there was a significant difference between the health care workers and the general population in this regard and this difference would be quite natural as the health care workers have more opportunities to explore the information about the vaccine. The results of a global survey published in Nature Medicine in October 2020 estimating the vaccine acceptance rates among the general population of various countries including India, showed that the acceptance rate was highest in China (88.62\%) and lowest in Russia (54.85\%). In India and the US, the acceptance rates were $74.5 \%$ and $75.4 \%$ respectively. ${ }^{8}$ In our study, the acceptance rate was $56.7 \%$ among the health care workers and $70.2 \%$ among the general population. Though the vaccine acceptance was similar in general population, it was low among the health care workers. On 26th January 2021, the results of a survey conducted in India among the general population in New Delhi, published in India Today, showed that $69 \%$ of Indians were hesitant to take the vaccine initially in the first week of January but when the same survey was repeated after 3 weeks, the hesitancy rate was reduced to $60 \%$. The acceptance rate increased from $31 \%$ to $38 \%$ within 3 weeks. ${ }^{9}$ However, in our survey, the vaccine hesitancy was $43.3 \%$ and $29.8 \%$ in health workers and general population respectively and the acceptance rate was $70.2 \%$ in the general population and $56.7 \%$ in health care workers. The hesitancy rate was low and the acceptance rate was high in the present survey when compared to that survey. ${ }^{9} \mathrm{An}$ online survey conducted among doctors in Apollo hospital, Chennai on 16th December 2020 showed that only $45 \%$ of the doctors were ready to accept the vaccine. ${ }^{7}$ In our survey, the vaccine acceptance rate among health care workers was $56.7 \%$. Though the acceptance has increased as compared to the previous surveys, still health care workers are more hesitant to get vaccinated as compared to the general population. The reluctance among the health care workers who have the responsibility of creating awareness to the public about the importance of vaccination, would be likely to influence the immunisation against COVID-19, as they themselves were not confident in receiving the vaccine. The overall vaccine acceptance rate was $80 \%$, however, only $30 \%$ of the respondents among these were ready to accept the vaccine immediately after availability. $51 \%$ of the participants wanted to delay the vaccine shot by 1 to 6 months and $19 \%$ were not sure when to get vaccinated. This delay in getting vaccination might affect the success of the immunisation programme against COVID-19. When we probed into the reasons for the hesitancy in getting vaccinated, most of the participants were doubtful about the vaccine's safety followed by their concern regarding 
its efficacy. Some participants were of the opinion that there was a lack of transparency regarding the clinical trials of vaccines in India. One of the limitations with COVID-19 vaccines is that the long-term efficacy has not been established as the vaccines have been developed in a short span and the clinical trials are still ongoing. Vaccine hesitancy poses a major challenge in the successful implementation of vaccination programmes in the control of infectious diseases like COVID-19. This could hinder the global efforts in controlling the current pandemic. Herd immunity that could be achieved through vaccination is very important in addition to other preventive measures in combating COVID-19. Hence, the government and nongovernmental agencies should take more efforts in gaining trust among the people ensuring that the available vaccines are safe and effective.

\section{Conclusion}

The results of this online survey show that the acceptance rate of the COVID-19 vaccine was more among the general population $(70.2 \%)$ as compared to health care workers $(56.7 \%)$ and the hesitancy rate was $43.3 \%$ among the health care workers and $29.8 \%$ among the general population. The reasons for vaccine hesitancy were fear of harmful effects, doubtful efficacy, lack of transparency in clinical trials, and fast track vaccine development.

\section{Acknowledgement}

The authors thank the Chettinad Academy of Research and Education for supporting them in conducting this online survey.

\section{Sources of Funding: None \\ Conflict of Interest: None \\ References}

1. World Health Organization [Internet]. WHO Coronavirus (COVID-19) Dashboard; [cited 2021 Feb 28]. Available from: https://covid19.who.int/

2. Krammer F. SARS-CoV-2 vaccines in development. Nature. 2020 Oct;586(7830):516-27. [PubMed] [Google Scholar]

3. U.S. Food \& Drug Administration [Internet]. PfizerBioNTech COVID-19 Vaccine. Available from: https:// www.fda.gov/emergency-preparedness-and-response/ coronavirus-disease-2019-covid-19/pfizer-biontechcovid-19-vaccine

4. U.S. Food \& Drug Administration [Internet]. FDA Takes Additional Action in Fight Against COVID-19 By Issuing Emergency Use Authorization for Second COVID-19 Vaccine. Available from: https://www.fda.gov/newsevents/press-announcements/fda-takes-additionalaction-fight-against-covid-19-issuing-emergency-useauthorization-second-covid
5. Fisher KA, Bloomstone SJ, Walder J, Crawford S, Fouayzi H, Mazor KM. Attitudes toward a potential SARS-CoV-2 vaccine: a survey of US adults. Ann Intern Med. 2020;173(12):964-73. [PubMed] [Google Scholar]

6. Freeman D, Loe BS, Chadwick A, Vaccari C, Waite F, Rosebrock L, Jenner L, Petit A, Lewandowsky S, Vanderslott S, Innocenti S, Larkin M, Giubilini A, Yu LM, McShane H, Pollard AJ, Lambe S. COVID-19 Vaccine Hesitancy in the UK: The Oxford Coronavirus Explanations, Attitudes, and Narratives Survey (OCEANS) II. Psychol Med. 2020 Dec 11;1-34. [PubMed] [Google Scholar]

7. The Hindu [Internet]. Coronavirus | Study warns of COVID-19 vaccine hesitancy. Available from: https:// www.thehindu.com/news/national/coronavirus-studywarns-of-covid-19-vaccine-hesitancy/article33348861. ece

8. Lazarus JV, Ratzan SC, Palayew A, Gostin LO, Larson HJ, Rabin K, Kimball S, El-Mohandes A. A global survey of potential acceptance of a COVID-19 vaccine. Nat Med. 2021; 27(2):225-8. [PubMed] [Google Scholar]

9. India Today [Internet]. $60 \%$ polled Indians still hesitant towards Covid-19 vaccine, shows survey. Available from: https://www.indiatoday.in/coronavirus-outbreak/ vaccine-updates/story/sixty-percentage-indians-stillhesitant-covid-19-vaccine-survey-1762749-2021-01-26 